\title{
Deshidroxilaciones y efectos Ostwald ripening en pizarras de techar
}

\author{
J. GARCIA-GUINEA ${ }^{1}$, v. CARDENES ${ }^{1}$, V. CORRECHER ${ }^{2}$, A. DELGADO ${ }^{2}$, M. LOMBARDERO ${ }^{3}$, J. C. BARROS ${ }^{4}$ \\ ${ }^{1}$ Museo Nac. Ciencias Naturales. Jose Gutierrez Abascal 2. Madrid 28006 \\ ${ }^{2}$ CIEMAT. Av. Complutense 22. Madrid 28040 \\ ${ }^{3}$ Instituto Tecnológico Geominero. C/ Rios Rosas 23. Madrid 28003 \\ ${ }^{4}$ Pizarras SAMACA S.A. El Trigal. Sobradelo de Valdeorras. Orense 32337
}

La pizarra solo exfolia en húmedo y cuando se ha mantenido en húmedo desde la cantera. Su irreversible secado, podría explicarse por cerramientos de porosidad según procesos Ostwald ripening de illita en los planos de pizarrosidad incluyendo transporte-salida de aguas con álcalis. La pizarra de techar presenta distribución continua de trampas de termoluminiscencia con acumulación de máximos alrededor de los $300^{\circ} \mathrm{C}$ que podría explicarse por formación-destrucción de centros [AlO ${ }_{4} /$ alcali $]^{+}$y $\left[\mathrm{AlO}_{4}\right]^{\circ}$. La evolución convergente de las curvas de termoluminiscencia de precalentados progresivos, hacia los $475^{\circ} \mathrm{C}$, coincide con los teóricos máximos térmicos de deshidroxilación. El secado aleatoriamente produce hinchamiento de láminas (001) y coalescencia de microcristales, habiéndose detectado grandes deformaciones del pico $10 \AA ̊$ de la illita-clorita mediante barridos isotermales (p.e., a $95^{\circ} \mathrm{C}$ ) de difracción de rayos $\mathrm{X}$ bajo estimulación termofotónica y control electrónico de temperatura. Estas grandes anomalías del pico de la cristalinidad de la illita en alícuotas a bajas temperaturas desaconsejan el uso petrológico del índice de Kubler difractando en el plano de exfoliación de micro-mono-bloques.

\section{Palabras clave: Ostwald ripening, pizarra, deshidroxilación, illita, termoluminiscencia}

\section{Dehydroxylation and Ostwald ripening effects in roofing slates}

Roofing slates only open in wet and when they are maintained in wet from quarry. Their irreversible drying could be explained by the closure of porosity following Ostwald ripening processes of illite along the slaty cleavage interface-interphases along with the leaking out water-alkali solutions. Roofing slate drying randomly produces swelling of layers and crystallite coalescence. They have been detected huge deformations of the illite-chlorite XRD $10 \AA$ peak by isothermal scanning (i.e., $95^{\circ} \mathrm{C}$ ) of XRD under thermo-photonic stimulation and electronic control of temperature. These large anomalies of the illite crystallinity peak working with aliquots of slate prevent against the petrological use of the Kubler index using the slaty cleavage of micro-mono-blocks. The slate also shows a continuous distribution of thermoluminescence traps with maxima concentration in $300^{\circ} \mathrm{C}$, which could be attributed to the creation-annihilation of $\left[\mathrm{AlO}_{4} /\right.$ alcali] ${ }^{+} \mathrm{y}[\mathrm{AlO} 4]^{\circ}$ centres. The convergent trend of thermoluminescence glow curves of preheated aliquots, circa $475^{\circ} \mathrm{C}$, coincides with the theoretical maxima of dehydroxylation temperature

Key words:Ostwald ripening, slate, dehydroxilation, illite, thermoluminescence

\section{INTRODUCCION}

La pizarra se forma en la naturaleza por metamorfismodiagénesis (aumento de presión y temperatura) de acumulaciones sedimentarias de arcillas. Desde el siglo pasado se sabe que la pizarras exfolian debido a que contienen minerales micáceos (filosilicatos). Están formadas por filosilicatos sedimentarios (granos detríticos) que se pueden observar por microscopía óptica y scanning de barrido (SEM) (tamaños alrededor de 10 micrómetros) y por filosilicatos neoformados (granos autigénicos) que solamente son detectables por microscopía electrónica de transmisión (TEM) (tamaños alrededor de 10 manómetros). España (Orense) es el primer productor mundial de pizarra de techar, siendo su fisibilidad o capacidad de ser hendida o exfoliada para obtener tejas ligeras y planas, la principal cualidad industrial exigida al producto comercial (1). La pizarra presenta otra particular propiedad: solo exfolia en húmedo y habiendo sido mantenida en húmedo desde la cantera. Sin embargo, abre mal cuando ha sufrido desecación, aunque, a posteriori, se intente rehidratar.
Se trata de un considerable problema tecnológico porque implica mucho movimiento industrial de contenedores con agua para mantener la humedad de los bloques de pizarra. Teniendo en cuenta que no existen explicaciones sencillas para este fenómeno, se está realizando un estudio de los mecanismos de desecación y deshidroxilación de las pizarras por: a) DTA-TG, b) Termoluminiscencia (Risø) y c) SOSXRD (Simultaneous Optical and Stimulated X-Ray Diffraction). Estas pizarras de techar fueron caracterizadas previamente por microscopías y termoluminiscencia espectral de alta sensibilidad (2).

La presencia de álcalis $\left(\mathrm{K}^{+}, \mathrm{Na}^{+}\right)$y aguas (hidroxilos, moléculas adsorbidas, y absorbidas) en una masa policristalina (illita, clorita, cuarzo) con interfases y pequeños tamaños de cristal, configura un sistema reactivo muy dinámico desde temperatura ambiente; por esta razón, han existido muchas teorías, observaciones y experimentos para explicar el origen geológico de la pizarrosidad. Por ejemplo, las hipótesis más 
recientes (3) explican la formación de la pizarra destacando los procesos mecánicos (rotación y/o plegado de granos) ó los procesos químicos (disolución y nuevo crecimiento de granos, -efecto Ostwald ripening-). De acuerdo con estos últimos datos (3), resulta muy difícil separar los papeles que juegan las energías térmicas y mecánicas en la formación de la pizarrosidad. Por esta razón, en la formación de la pizarrosidad intervienen la temperatura y la tectónica y ambas contribuyen a la energía total del sistema. El mecanismo de deformación dominante queda definido por la energía total del sistema, en regiones de metamorfismo de bajo grado y escaso tectonicismo, las transformaciones mecánicas requieren menos energía que los cambios químicos. Los procesos mecánicos (micropliegues y rotación de granos) se producen mejor en medios de baja energía, mientras que los procesos químicos (disolución de granos y neocristalizaciones) se producen mejor en medios mas energéticos.

En filosilicatos dispersos (illita, clorita), se puede asumir que los principales modificadores de la geometría del pico de difracción de rayos X (DRX) a $10 \AA$ de la clorita-illita son el tamaño de cristal y el hinchamiento de las capas 001 (4-5). Los mecanismos de deshidroxilación de la moscovita-illita son conocidos, cabiendo destacar que hacia $\operatorname{los} 475^{\circ} \mathrm{C}$, la red cristalográfica expande doble según el eje c (001) que según los otros ejes (6-7). Finalmente, hay que recordar que en la illita también están descritos los fenómenos Ostwald ripening (8-9) que consisten en el crecimiento y disolución simultáneos de partículas en un medio (p.ej., pizarra), tendiendo a minimizar la superficie de energía libre por disolución de cristales pequeños y crecimiento de otros mayores, según un mecanismo de transporte de masa en disolución (p.e. salida de agua de secado en una pizarra).

Los cambios geométricos de las reflexiones basales de DRX de illita se han utilizado desde la década de los sesenta (10) como indicadores geológicos termobáricos; el índice de cristalinidad de la illita o índice de Kübler (IC) relaciona la anchura del pico de DRX a $10 \AA$ (medido a media altura) con la cristalinidad de la illita. El valor de IC disminuye con el aumento de cristalinidad de la illita coincidiendo con aumentos de diagénesis y/o metamorfismo (11). Las variables que afectan al valor de IC incluyen temperatura, presión de fluidos, tectónica de cizalla, duración de la diagénesis de enterramiento, condiciones metamórficas, litología, composición de la illita, presencia de otros filosilicatos, preparación de la muestra y condiciones instrumentales. Los efectos de muchas de estas variables están deducidos más de correlaciones con la naturaleza e historia de las rocas estudiadas que a partir de observaciones directas de la química, textura y estados estructurales de la illita en comparación con su IC. Hemos considerado utilizar una modificación del método BWA (Bertaut-WarrenAverbach) recientemente propuesta (12) para medir los tamaños de dominios de difusión coherentes y las tensiones en los minerales de la pizarra por DRX. Sin embargo tuvimos que descartar este método porque, previamente, hemos visto que sus estudios no funcionan en muestras de rocas en bloque. Estos mismos autores (9) utilizan análisis de Fourier y TEM para obtener tamaños de espesor de cristalito según $c^{*}$, asumiendo que el tamaño de cristalito y el hinchamiento de las capas son las principales contribuciones a la geometría del pico de DRX $10 \AA$, sin embargo, especifican esto solo se puede afirmar para partículas dispersas.

Más tarde, en este trabajo, difractando placas alícuotas de pizarra pulida según (001) a bajas temperaturas (por debajo de $100^{\circ} \mathrm{C}$ ) hemos detectado espectaculares anomalías en geo- metrías del pico $10 \AA ̊$. Este hecho, nos está llevando a pensar que, efectivamente, el tamaño de cristalito y el hinchamiento de las capas podrían ser las principales contribuciones a la geometría del pico a $10 \AA$ de DRX. Pero también hay que recordar, que se trataría de un sistema muy dinámico donde unas bajas temperaturas de secado y evaporación de aguas de adsorción y absorción podrían modificar estos factores mediante mecanismos Ostwald ripening a lo largo de los planos de interfases. Esta línea de razonamiento, de paso, podría explicar la irreversibilidad de las pérdidas de agua por secado y las disminuciones de fisibilidad por secado observadas en los talleres industriales de hienda y corte de pizarra.

Solo ha sido posible hacer estas observaciones utilizando el prototipoSOSXRDrecientementeconstruidoenelDepartamento de Geología del Museo Nacional de Ciencias Naturales de Madrid, que permite hacer difracción de rayos $\mathrm{X}$ en automático, bajo radiación termal simultánea in situ con control electrónico de temperatura, con el objetivo de imitar las condiciones naturales de solarización-desecación de la pizarra.

\section{PROCEDIMIENTO EXPERIMENTAL}

En Sobradelo de Valdeorras (canteras de SAMACA, Orense) se extrajo un bloque (5 TM aprox.) de pizarra de alta fisibilidad en condiciones de humedad natural mediante taladros e hilo de diamante. Seguidamente, en la planta industrial, se cortó un bloque de $6 \mathrm{Kg}$ con disco de diamante y se guardaron dos alícuotas, una seca fuera, y otra en un contenedor con agua. La mineralogía de la muestra fue estudiada previamente por microscopías y difracción de rayos $\mathrm{X}$.

Para determinar al mismo tiempo las variaciones de masa de la pizarra en función del tiempo-temperatura (termogravimetría, TG) con una rampa térmica de $4 \mathrm{~K} /$ minuto y las diferencias de temperatura entre la pizarra y el corindón de referencia (análisis térmico diferencia, ATD), se utilizó un analizador simultáneo de ATD-TG marca Netzsch (Mod. 409/3/410/D) con un sistema de pesada con compensación electromagnética en un horno (hasta $1600^{\circ} \mathrm{C}$ ) en atmósfera de nitrógeno.

Para analizar las emisiones termoluminiscentes en el azul de alícuotas de pizarra con diferentes temperaturas de precalentamiento (desde $250^{\circ} \mathrm{C}$ hasta $425^{\circ} \mathrm{C}$ en intervalos de $25^{\circ} \mathrm{C}$ en $25^{\circ} \mathrm{C}$ ), se preparó una batería de 24 discos de aluminio con alícuotas de $5.0 \pm 0.1 \mathrm{mg}$ de pizarra en un sistema automático de TL/OSL automatizado modelo TL-DA-12 desarrollado por Risø National Laboratory de Roskilde, Dinamarca (12). Este lector consta de una unidad principal con portamuestras, horno, irradiador provisto de una fuente de Sr / Y-90 cuya tasa de dosis es $0.025 \mathrm{~Gy} / \mathrm{s}$ y un tubo fotomultiplicador EMI modelo THORN 9635QA. Todas las medidas se efectuaron en presencia de nitrógeno y a una velocidad de calentamiento de $5 \mathrm{~K} / \mathrm{s}$

Las medidas de caracterización previas de DRX fueron efectuadas en un difractómetro Siemens D-5000 con radiación $\mathrm{CuK} \alpha$. Los difractogramas fueron obtenidos desde $2^{\circ} 2 \theta$ hasta $64^{\circ} 2 \theta$ en saltos de $0.020^{\circ}$ con 6 s por escalón. Las medidas de anisotropía de absorción y difracción de rayos $X$ en diferentes orientaciones espaciales de bloques de pizarra se hicieron siguiendo el método Chen (1991) (15). Para el estudio de DRX con radiación termal simultánea y control de temperatura, en un difractómetro Philips PW-1710 se instaló: a) un termopar y una lámpara halógena dentro de la cámara de difracción, b) un controlador de temperatura Eurotherm y 
c) unos tiristores para regular el voltaje en modo de ángulo de fase. El bucle de control automático de voltaje-temperatura se hizo por el método proporcional-integral-derivativo (PID). El sistema permite obtener difractogramas secuenciales bien en forma isotermal automática o bien manualmente con rampa termal.

\section{RESULTADOS Y DISCUSION}

Al microscopio, la muestra de pizarra contiene, en orden de importancia, illita, micas fengíticas sodico-potásicas $2 \mathrm{M}_{1}$, algo de paragonita, clorita, cuarzo, feldespatos detríticos, ilmenita, rutilo, apatito, cloritoide y turmalina. Texturalmente, está compuesta de partículas de tamaño limo de cuarzo detrítico formando pequeños micropliegues, feldespatos, apilamientos mica-clorita, moscovita e ilmenita en una matriz lepidoblástica recristalizada de micas blancas, clorita y lentes de cuarzo. Todo ello mostrando una fuerte orientación preferencial. La turmalina, el rutilo y el apatito están dispersos en la matriz y frecuentemente también muestran orientación cristalográfica preferencial. La pizarra tiene solo escasos apilamientos de clorita-mica dispersos en una matriz de filosilicatos de grano
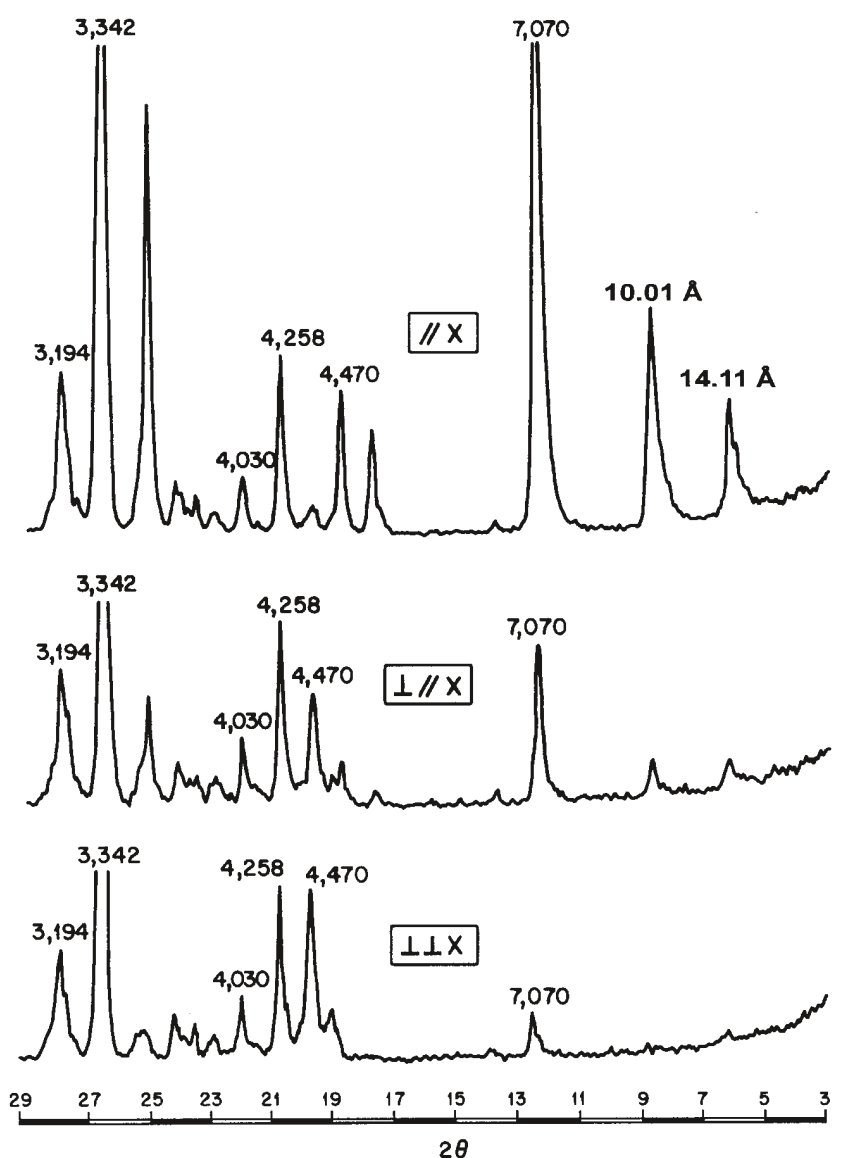

FIGURA 1.- Difractogramas de un bloque de pizarra de techar orientado segun sus tres orientaciones principales: arriba: Plano de apertura de la pizarrosidad (/ / X) mostrando mayores picos (001) de la illita y clorita $(10.01 \AA$ y $14,11 \AA$ ). medio: Plano perpendicular a la pizarrosidad $(\perp / / X)$ mostrando pequeños picos 001 de moscovita $y$ clorita y ABAJO: Plano $(\perp \perp \mathrm{X})$ no presentando los picos $10.01 \AA \mathrm{y}$ $14,11 \AA$ de illita y clorita. muy fino altamente orientados, que definen los planos $S_{1}$. Los contactos intergranulares muestran relaciones de deformación. Los apilamientos están fuertemente deformados con relaciones axiales de 1:4 a 1:5. Tienen tamaños de hasta 60×250 $\mu \mathrm{m}$, pero normalmente son de 20x100 $\mu \mathrm{m}$. Los granos de cuarzo detrítico también están ampliamente dispersos, fuertemente deformados $y$, normalmente, tienen un tamaño de 10x30 $\mathrm{mm}$. Los granos de feldespatos potásicos tienen tamaños similares y solo se presentan ocasionalmente. El tamaño del cuarzo y feldespato más común es el de limo, mientras que los apilamientos de clorita-mica alcanzan tamaños de arena muy fina. También aparecen micro-porfiroblastos rectangulares de cloritoide orientados según el plano $\mathrm{S}_{1}$, alcanzando tamaños de hasta 150x15 $\mu \mathrm{m}$. Ocasionalmente, aparecen en secciones transversales con tamaños de hasta $100 \times 25 \mu \mathrm{m}$; en estos casos desarrollan contactos de presión. La fracción $<2 \mu \mathrm{m}$ fue identificada por DRX y en orden de abundancia decreciente es mica sódico-potásica $2 \mathrm{M}_{1}$, clorita, paragonita, cuarzo, albita y rutilo. El cloritoide y el feldespato potásico no se detectan en esta fracción. El tamaño y la frecuencia de los apilamientos mica-clorita y los micro-porfiroblastos no orientados de cloritoide influyen sobre el espesor de las placas que se pueden obtener de esta pizarra. La muestra tiene un índice de Kubler de 0,29 , pero este valor está exagerado por la presencia de mica sódico-potásica y una discreta cantidad de paragonita. El parámetro de celdilla b de las micas blancas, que es $9,01 \AA$, apunta hacia unas facies metamórficas de baja presión.

Además de estos estudios previos de caracterización de fases mineralógicas, también por DRX (polvo cristalino), se hicieron ensayos de anisotropía de difracción y absorción de rayos $\mathrm{X}$ según las orientaciones espaciales de la pizarra (Figura 1). Evidentemente, el plano de apertura de la pizarrosidad (/ / X), o plano $X_{1} X_{2}$ de lineación de Chen, muestra los mayores picos (001) de la illita y clorita confirmando la orientación 001 generalizada de los filosilicatos. Este plano contiene la lineación geológica. El plano perpendicular a la pizarrosidad $(\perp / / \mathrm{X})$, o $\mathrm{X}_{1} \mathrm{X}_{3}$ slaty cleavage de Chen, muestra pequeños picos 001 de moscovita y clorita y por último el plano $(\perp \perp X)$ o plano $\mathrm{X}_{2} \mathrm{X}_{3}$ de Chen no presenta los picos $10.01 \AA ̊$ y $14,11 \AA ̊$ de la illita y clorita.

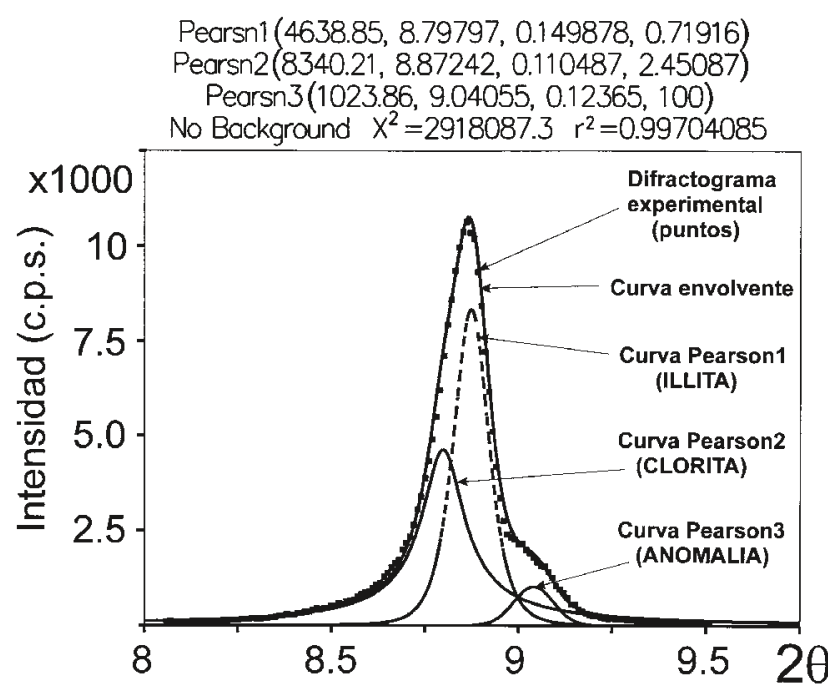

Figura 2.- Deconvolución con función Pearson utilizando el programa Peakfit. La curva envolvente del pico de difracción de $10 \AA$ contiene tres curvas: IZQUIERDA: Clorita, MEDIO: Illita, IZQUIERDA Anomalía de hinchamiento y coalescencia de granos producida por solarización artificial (Sistema SOSXRD). 
DRX ISOTERMAL $\left(95^{\circ} \mathrm{C}\right)$
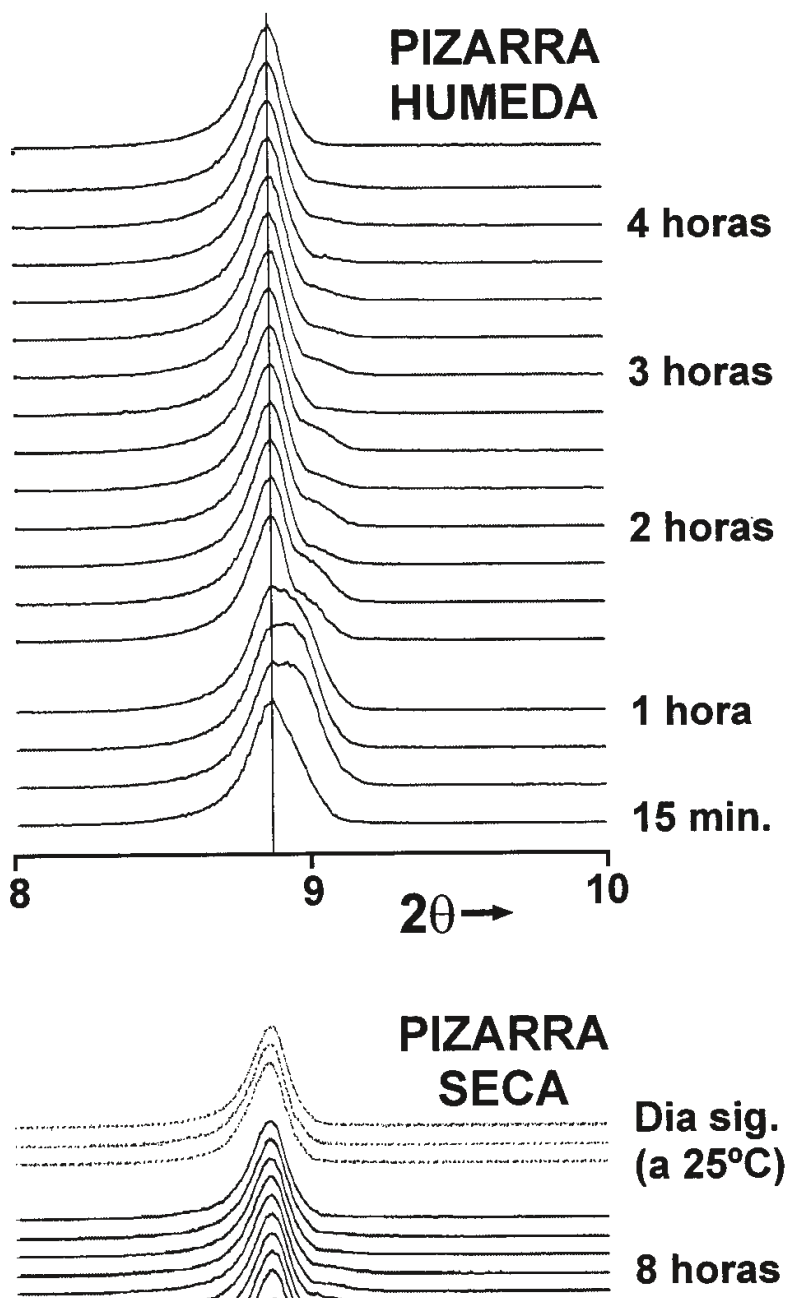

7 horas

6 horas

5 horas

4 horas

3 horas

2 horas

1 hora

$15 \mathrm{~min}$.

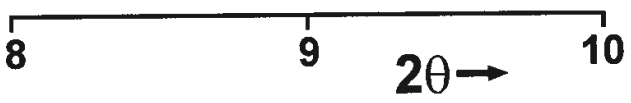

FIGURA 3.- Análisis isotermales $\left(95^{\circ} \mathrm{C}\right)$ de DRX bajo irradiación termofotónica y control térmico (SOSXRD) de dos alícuotas monobloque basal de pizarra, una húmeda y otra seca. Nótese la gran deformación del pico $10 \AA ̊$ illita-clorita y la diferencia de tiempos de desarrollo. Además, su presencia en otras alícuotas es aleatorio, a veces, no aparece.
Los análisis TG de la pizarra tanto la seca como la húmeda (Figura 4), mostraron unas pérdidas de un $0.76 \%$ en el intervalo $20^{\circ} \mathrm{C}-100^{\circ} \mathrm{C}$. Estos resultados, igualan las aguas de absorción y adsorción de ambas muestras, quizás, debido a la misma baja porosidad. Sin embargo, si se considera el intervalo $20^{\circ} \mathrm{C}-400^{\circ} \mathrm{C}$, la seca pierde $1,38 \%$ y la húmeda $1,97 \%$. En el intervalo $400^{\circ} \mathrm{C}-600^{\circ} \mathrm{C}$, la seca pierde $1,23 \%$ y la húmeda $0,97 \%$, es decir, deshidroxila mejor, la pizarra seca. Una forma de entender estos datos podría ser considerando las interferencias álcalis $(\mathrm{Na} / \mathrm{K})$ - aguas $\left(\mathrm{OH}, \mathrm{O}, \mathrm{H}_{2} \mathrm{O}\right)$ durante los procesos de deshidroxilación.

La figura 5 de termoluminiscencia (TL) de la pizarra, muestra una clásica distribución continua de trampas. Se representan curvas de TL natural (intensidad por mg en función de la temperatura en grados centígrados obtenidas a partir de muestras de pizarra seca sin irradiar con diferentes precalentamientos (desde $250^{\circ} \mathrm{C}$ hasta $425^{\circ} \mathrm{C}$ en intervalos de $25^{\circ} \mathrm{C}$ en $25^{\circ} \mathrm{C}$ ). En la estructura de las curvas destaca la presencia inicial de dos máximos, que a medida que sufren calentamientos progresivamente mayores se produce un desplazamiento del primer máximo $\left(310^{\circ} \mathrm{C}\right)$ hacia zonas de alta temperatura. Mientras el pretratamiento térmico no alcanza $340^{\circ} \mathrm{C}$ el segundo máximo no se ve afectado, pero, pretratamientos térmicos más altos hacen que el comportamiento del máximo a $370^{\circ} \mathrm{C}$ sea similar al observado anteriormente en el primero. Este movimiento monotónico de los máximos se asocia a un progresivo vaciado de trampas llenas que se encuentran en niveles energéticos muy próximos. Y se denomina distribución continua de trampas. En consecuencia, los picos de termoluminiscencia no estarían definidos por el proceso típico de termoluminiscencia donde la temperatura del máximo depende de los parámetros de atrapamiento (i.e. profundidad de la trampa -E- y el factor de frecuencia -s-) que se define por una ecuación general del tipo:

$$
\beta E / k T^{2}{ }_{m}=n^{b} \operatorname{sexp}\left(-E / k T_{m}\right)
$$

(donde $\beta$ es la velocidad de calentamiento, $\mathrm{n}$ es el número de portadores de carga, $\mathrm{b}$ es un valor determinado empíricamente conocido como orden de cinética, s depende de la frecuencia de vibración térmica y del coeficiente de transmisión

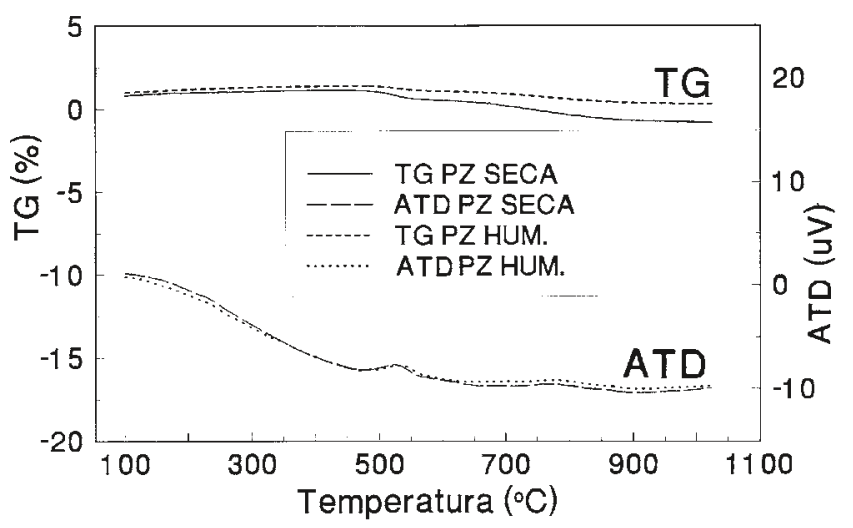

FIGURA 4.- Análisis ATD-TG de microbloques alícuotas de pizarra húmeda y seca. El pico de deshidroxilación se desplaza ligeramente hacia mayores temperaturas $\left(525^{\circ} \mathrm{C}\right)$ respecto del teórico $475^{\circ} \mathrm{C}$ por las diferentes rampas térmicas (ATD-TG $4^{\circ}$ / minuto). 
y el último término del segundo miembro de la ecuación es un factor de entropía); sino que el proceso vendría dado por una distribución de tiempos de vida $(\tau)$ de los electrones en las trampas definido por (14):

$$
\tau=(1 / s) \exp (E / k T)
$$

Por otra parte, en estructuras análogas de filosilicatos (caolinita), se ha observado un comportamiento muy similar de distribución continua de trampas, habiéndose asociado las emisiones fotónicas con procesos de deshidroxilación y cambios bruscos en las impedancias eléctricas (16).

Con este precedente, estas curvas experimentales también podrían asociarse a deshidroxilaciones ya que la secuencia de las curvas de TL de pizarras precalentadas muestra dos temperaturas clave: a) alrededor de $475^{\circ} \mathrm{C}$ coincidiendo con la temperatura de deshidroxilación de la illita (6) y b) alrededor de $300^{\circ} \mathrm{C}$ mostrando muchas coincidencias con los centros de emisión de luminiscencia $\left[\mathrm{AlO}_{4} / \text { alcalii }\right]^{+}$y $\left[\mathrm{AlO}_{4}\right]^{\circ}$ observados en otros aluminosilicatos con álcalis (feldespatos) (17-18). Estos centros de emisión, se forman-destruyen por procesos de autodifusión iónica de álcalis (inducida termalmente) a lo largo de las interfases cristalográficas. Al analizar los fenómenos fototermales en la pizarra, conviene tener en cuenta que: a) calentando la illita, hacia los $475^{\circ} \mathrm{C}$, expande doble segun $\mathrm{c}^{*}$ que según los otros ejes, b) los planos 001 marcan las rutas de deshidroxilación y salidas de agua, c) la deshidroxilación de la illita debe cumplir el principio de compensación valencia electrostática de Pauling, d) por razones de cinética de interacciones átomo-átomo, la deshidroxilación debe ser temporal respecto al incremento de la temperatura, perdiéndose unos hidroxilos antes que otros, e) la curva TG indica que la deshidroxilación ocurre durante un amplio intervalo termal, f) en la termo-deshidroxilación de la illita también hay que incluir el movimiento de iones de potasio, g) el fenómeno Ostwald ripening está comprobado por TEM y DRX-BWA en puntas de apilamientos de illita (9), h) la moscovita se degrada a illita a temperatura ambiente en medios acuosos, i) la pizarra es un sistema complejo y altamente reactivo por el pequeño tamaño de sus partículas, elevada superficie de interfases proximas, presencia de agua y álcalis.

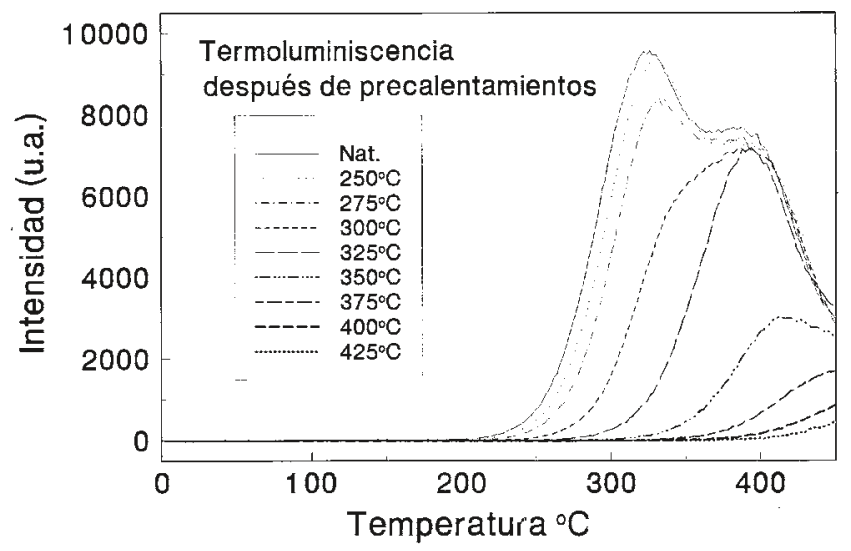

FIGURA 5.- Termoluminiscencia. Distribución continua de trampas de curvas de alícuotas con diferentes temperaturas de precalentamiento. Temperatura $310^{\circ} \mathrm{C}$ : centros de emisión de luminiscencia $\left[\mathrm{AlO}_{4} / \text { alcali }\right]^{+}$ y $\left[\mathrm{AlO}_{4}\right]^{\circ}$. Temperatura $475^{\circ} \mathrm{C}$ : Deshidroxilación de la illita.

\section{CONCLUSIONES}

Añadiendo estos experimentos TL, ATD-TG y SOSXRD al conocimiento existente sobre deshidrataciones-deshidroxilaciones de pizarras e illitas se puede tener una visión mas completa de varias propiedades físicas sorprendentes de la pizarra de techar. La pizarra, que es un cuerpo negro, en España, en verano y al sol, podría alcanzar en algunos momentos $\operatorname{los} 70^{\circ} \mathrm{C}$ - se están instalando tiny-talks de temperatura y humedad en taladros sellados para comprobarlo-. Por este motivo, el propio secado solar puede suponer un proceso mini-hidrotermal con fenómenos Ostwald ripening en los planos de pizarrosidad implicando disoluciones-recristalizaciones con sellado de porosidad y soldado de muchas posibles interfases de exfoliación industrial de pizarra. La distribución continua de trampas de termoluminiscencia con temperaturas críticas alrededor de $\operatorname{los} 300^{\circ} \mathrm{C}$ podría explicarse por formación-destrucción de centros $\left[\mathrm{AlO}_{4} / \text { alcali] }\right]^{+}$y $\left[\mathrm{AlO}_{4}\right]$ o siguiendo las teorías de Martini. La evolución convergente de las curvas de TL de precalentados progresivos hacia los $475^{\circ} \mathrm{C}$ coincide con los máximos térmicos de deshidroxilación que puede ser compatible con fenómenos Ostwald ripening de disolución-cristalización de illita, fundamentalmente en las interfases cristalográficas y mineralógicas de la pizarrosidad. Los espectaculares y aleatorios cambios de geometría bajo acción del SOSXRD hacia los $95^{\circ} \mathrm{C}$, muestran grandes heterogeneidades, muy posiblemente debido a que las diferencias espaciales de las interfases condicionan diferentes hinchamientos y coalescencias de granos. Evidentemente, esto supone la imposibilidad de medir índices geológicos de Kubler en bloques orientados de metapelitas.

\section{AGRADECIMIENTOS}

El presente trabajo ha sido financiado con los proyectos: 1FD97-0959-C03-01 del Plan Nacional de Investigación Científica y Desarrollo Tecnológico y PB 98-0501-B de la Dirección General de Investigación Científica y Técnica. Asimismo, tenemos que agradecer a Rafael González Martín las medidas de difracción de rayos X. Esperanza Menéndez realizó las medidas de ATD-TG en el Instituto Torroja (CSIC). José María Barrenechea caracterizó las fases mineralógicas por A.O. y polvo cristalino de las muestras pretratadas.

\section{BIBLIOGRAFIA}

1. J.Garcia Guinea, M. Lombardero, B. Roberts, J. Taboada. "Spanish roofing slate deposits". Trans. Instn. Min. Metall. (Sect. B: Appl. Earth. Sci.), 106, 205214 (1997).

2. J. Garcia Guinea, M. Lombardero, B. Roberts, J. Taboada, A. Peto. “Mineralogía y microestructura de la pizarra de techar: comportamiento termoóptico y fisibilidad". Materiales de Construcción 48 (251), 37-48 (1998).

3.B.A. Van-Der-Pluijm, H. Nei-Che, R.J. Merriman,. “Contradictions of slate formation resolved?". Nature, 392, 348, (1998).

4. H. Kodama. "Crystal distortion of sericite." Clay Science 2, 121-131 (1965)

5. W.T.Jiang, D.R. Peacor, P. Arkai, M. Toth, J.W. Kim. “Tem and XRD determination of crystallite size and lattice strain as a function of illite crystallinity in pelitic rocks." J. Metam. Geol. 15, 267-281 (1997).

6. H.Takeda, B. Morosin. "Comparison of observed and predicted structural parameters of mica at high temperatures." Acta Crystall. B31, 2444-2452 (1975).

7. S.Guggenheim, Y.H. Chang, A.F. Koster, V.,Groos. “Muscovite dehydroxylation: High temperature studies." American Miner 72, 537-550 (1987).

8. A. Baronnet. "Ostwald ripening in solution . The case of calcite and mica". Estudios Geol. 38, 185-198 (1982). 
9. D.D. Eberl, J. Srodon. “Ostwald ripening and interparticle-diffraction effects for illite crystals". American Miner. 73, 1335-1345 (1988).

10. B. Kübler. "La cristallinité de l'illite et les zones tout à fait supérieures du métamorphisme". In: Etages tectoniques, pp.105-121. Colloque de Neuchatel 1966. A La Bacounière, Neuchâtel, Suisse. (1967).

11. M. Frey. "Very low-grade metamorphism of clastic sedimentary rocks." In Low Temperature Metamorphism. (ed. Frey, M.) pp.9-58. Blackie, Glasgow. (1987).

12. V.A. Drits,D.D. Eberl, J. Srodon. "XRD measurement of mean thickness, thickness distribution and strain for illite-smectite crystallites by BertautWarren-Averbach technique". Clays and Clay Miner. 46(1), 38-50 (1998).

13. L. Bøtter-Jensen y G.A.T.Duller. "A new system for measuring OSL from quartz samples." Nucl. Tracks \& Radiat. Meas. 20, 549-553 (1992).

14. M.Martini, G. Spinolo, A. Vedda, C. y Arena. "Phosphorescence and therma- lly stimulated luminescence of amorphous $\mathrm{SiO}_{2} . "$ Sol. Stat. Comm. 91(9)751756 (1994).

15. R. Chen. "Anisotropy of X Ray Absorption in a Slate: Effects on the Fabric and March Strain Determination." Jour. Geophys. Res. 96(B4), 6099-6105 (1991).

16. J. Garcia Guinea, V.Correcher, F.J. Valle-fuentes. “Thermoluminescence of Kaolinite." Radiat. Prot. Dosim. 84(1-4) 507-510, (1999)

17. M. Martini, A. Paleari, G. Spinolo, A. Vedda . "Role of [AlO4] $\infty$ centers in the $380 \mathrm{~nm}$ thermoluminescence of quartz." Phys Rev B, 52,138-142, (1995).

18. J. Garcia-Guinea, H.M. Rendell, L. Sanchez-Muñoz. "Luminescence spectra of alkali feldspars: some relationships between structural features and luminescence emission". Radiat Protect Dosim 66, 395-398, (1996).

19. J. Garcia-Guinea, P.D.Townsend, L.Sanchez-Muñoz, J.M. Rojo. “Ultravioletblue ionic luminescence of alkali feldspars from bulk and interfaces." Phys. Chem. Miner. 26 658-667, (1999). 